\title{
Multi-channel pseudo-random coding single-photon ranging and imaging
}

\author{
Di Wu (伍 狄) ${ }^{1}$, Lei Yang (杨 雷) ${ }^{1}$, Xiuliang Chen (陈修亮) ${ }^{1}$, Zhaohui Li (李召辉) ${ }^{1 *}$, and Guang Wu (吴 光) ${ }^{1,2}$ \\ ${ }^{1}$ State Key Laboratory of Precision Spectroscopy, East China Normal University, Shanghai 200241, China \\ ${ }^{2}$ Collaborative Innovation Center of Extreme Optics, Shanxi University, Taiyuan 030006, China
}

*Corresponding author: zhhli@|ps.ecnu.edu.cn

Received September 18, 2021 | Accepted November 2, 2021 | Posted Online November 19, 2021

\begin{abstract}
We demonstrate a multi-channel pseudo-random coding single-photon ranging system. A pseudo-random multiplexing technique is proposed, which realizes multi-channel pseudo-random ranging only by using one single-photon detector and processing circuit. Compared with the time division multiplexing technique, it will not reduce the maximum unambiguous range while increasing the number of the ranging channel. Eight-channel pseudo-random coding single-photon ranging was realized with the ranging accuracy better than $2 \mathrm{~cm}$. Moreover, photon counting imaging was realized through scanning the laser beams of the eight-channel pseudo-random ranging system. There is no crosstalk between channels, which is suitable for multi-beam long-distance single-photon Lidar.
\end{abstract}

Keywords: single-photon detection; pseudo-random modulation; laser ranging.

DOI: 10.3788/COL202220.021202

\section{Introduction}

Single-photon ranging attracted much attention in the field of vehicle-mounted Lidar $^{[1-3]}$, satellite-ground measurement ${ }^{[4]}$, and unmanned aerial vehicle $\operatorname{Lidar}^{[5,6]}$. Most single-photon ranging systems use the time-of-flight (TOF) method ${ }^{[7,8]}$. The time correlated single-photon counting (TCSPC) ${ }^{[9-11]}$ technique is widely used to extend the measurement sensitivity and precision, when the laser pulse energy remains unchanged. On the other hand, the multi-beam method is introduced for single-photon Lidar to improve the measurement speed ${ }^{[12-15]}$. The Leica SPL100 splits a $532 \mathrm{~nm}$ laser into $10 \times 10$ laser beams and receives the echo photons by a $10 \times 10$ single-photon detection array of photomultiplier tubes $(\mathrm{PMTs})^{[16]}$. It increases the sampling rate by increasing the number of laser beams. However, the detection array is complicated and expensive. In order to simplify the multi-beam detection system, researchers have proposed technologies such as time division multiplexing $(\mathrm{TDM})^{[17,18]}$ or repetition frequency multiplexing ${ }^{[19]}$ to improve the utilization of the detection system, so that one single-photon detector (SPD) and timing circuit can detect the echo photons of multiple beams at the same time. The TDM method uses multiple pulsed lasers with the same repetition frequency and different pulse delays. In order to distinguish the echo photons of each laser beam, it is necessary to ensure that the echo photons of the previous laser beams have been detected before the next laser beam is emitted. If the number of channels increases, the delays between the laser beams should be decreased, resulting in decreasing the maximum unambiguity range.

In this paper, we propose a pseudo-random (PR) multiplexing technique for multi-beam single-photon ranging and imaging. In the experiment, eight groups of PR codes were used to modulate eight lasers. Only one SPD and processing circuit were used to realize eight-channel ranging at the same time. The period of the PR code was $10 \mu \mathrm{s}$, and the maximum unambiguous range of all the ranging channels was $1.5 \mathrm{~km}$, which was not shortened with the increase of ranging channel. It is useful for longdistance multi-beam single-photon ranging and imaging.

\section{Generation of the Pseudo-Random Code}

PR numbers were generated by mathematical recursive formulas. The appearance of this group of numbers conformed to a certain probability distribution and can pass the corresponding randomness test ${ }^{[20]}$. In our PR ranging system, the PR numbers were generated in MATLAB by using the Mersenne twister algorithm, which has been widely used ${ }^{[21]}$. The PR code was improved according to the characteristics of single-photon detection. Eight groups of return to zero PR codes ${ }^{[22]}$ were generated. The PR code parameters are listed in Table 1. Each PR code contains 30 bits of ' 1 ', and at least 15 bits of ' 0 ' were inserted between two adjacent ' 1 '. The cross-correlation coefficient between each group of PR codes was less than 0.006, where the crosstalk between PR codes was very small. 
Table 1. PR Code Parameters.

\begin{tabular}{lc}
\hline Parameter & Value \\
\hline Code length & $5000 \mathrm{bit}$ \\
Code period & $10 \mu \mathrm{s}$ \\
Bit duration & $2 \mathrm{~ns}$ \\
Laser pulse period & $0.5 \mathrm{~ns}$ \\
Unambiguous range & $1500 \mathrm{~m}$ \\
\hline
\end{tabular}

\section{Multi-Channel PR Coding Single-Photon Ranging}

Figure 1 is the schematic diagram of the multi-channel PR coding single-photon ranging system. Eight groups of PR codes were stored in the field programmable gate array (FPGA). The FPGA called the PR codes and generated a short pulse of 2 ns for each bit ' 1 '. Then, the FPGA called 8 sets of PR codes to generate 8 sets of pseudo-random pulse sequences respectively to drive 8 distributed feedback (DFB) laser diodes, and additionally generated a single pulse signal with the same repetition frequency as the PR code as the synchronization signal of time-to-digital converter (TDC). The time resolution of the TDC was 160 ps. The wavelengths of the eight lasers were C18, C20, C22, C20, C34, C36, C38, and C44 (1542.73$1563.05 \mathrm{~nm}$ ) in the fiber communication C-band, respectively. The wavelengths were stabilized by temperature control of the DFB laser diodes. The lasers were coupled into an erbium-doped fiber amplifier (EDFA) through dense wavelength division multiplexing (DWDM). The lasers were collimated and passed through the small hole of a perforated mirror. The echo light was reflected by the perforated mirror and coupled into a fiber-pigtail InGaAs/InP avalanche photodiode (APD) SPD. We used a negative feedback InGaAs/InP APD for free-running

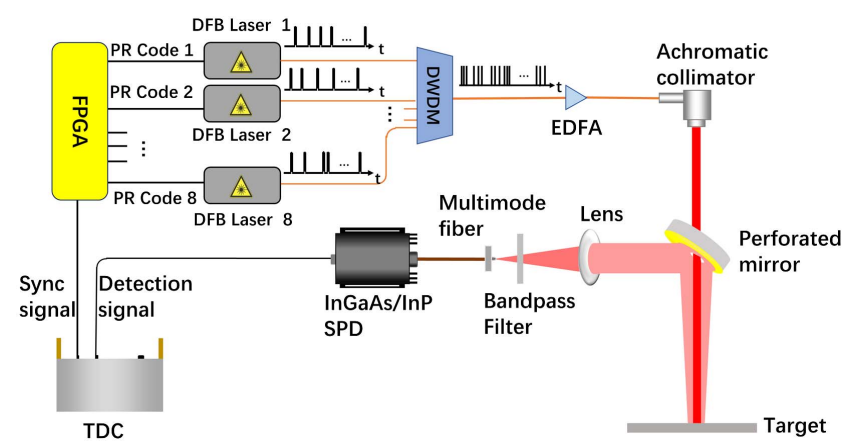

Fig. 1. Schematic diagram of the multi-channel PR coding single-photon ranging system. Achromatic collimator: output beam size is $12 \mathrm{~mm}$ in diameter, and full angle beam divergence is $0.02^{\circ}$ (Thorlabs RC12APC-P01). Perforated mirror: $D=50 \mathrm{~mm}$, and the diameter of the hole is $12 \mathrm{~mm}$. Focusing lens: $D=30.0 \mathrm{~mm}$, and $F=100.0 \mathrm{~mm}$. Bandpass filter: center wavelength is $1550 \mathrm{~nm}$, and the full width at half-maximum is $40 \mathrm{~nm}$ (Thorlabs FB1550-40). Multimode fiber: fiber core is $62.5 \mu \mathrm{m}$ in diameter. single-photon detection ${ }^{[23]}$. There was a $1 \mathrm{M} \Omega$ resistor in series with the anode of the APD chip. This APD was typically operated in passive quenching Geiger mode, and its afterpulse was effectively suppressed owing to the feedback resistor. As a result, a short dead time near-infrared SPD was obtained. The detection efficiency was $4.5 \%$ with the dark count of $2 \times 10^{5}$ counts per second, while the APD was cooling at $-30^{\circ} \mathrm{C}$. The dead time was about $200 \mathrm{~ns}$, with a maximum counting rate of $\sim 5.8 \times 10^{6}$ counts per second (cps).

The echo photons of all the lasers were detected by one singlepixel SPD at the same time. The TDC recorded the interval time between the synchronization signal and the detection events. In order to improve the signal-to-noise ratio (SNR), the TCSPC technique was used to count the echo signals of multiple repetition periods.

First, we tested the performance of single-channel PR coding single-photon ranging. The time distribution histogram of the detection events was obtained by TCSPC with the accumulated time of $1.0 \mathrm{~s}$, as shown in Fig. 2(b). The histogram of the detection events was a group of 62,500 bits data, while the resolution of the TDC was 160 ps within $10 \mu$ s, denoted as $X(t)$. The result of cross correlation between the PR code $Y_{n}(t)$ and the detection events was calculated as

$$
R_{X Y_{n}}(\tau)=\int_{0}^{m-1} X(t) Y_{n}(t+\tau) \mathrm{d} t
$$

where $m$ is the length of detection events, and $n$ is the channel number of the PR code. The flight time was obtained by calculating the cross-correlation peaks with the centroid $\operatorname{method}^{[24]}$.

As shown in Figs. 2(a)-2(e), most photon counts of the signal photons were larger than 20 . The noise counts less than 20 were filtered out before calculating the correlation function. As shown in Fig. 2(f), a high SNR cross-correlation peak was obtained when only DFB laser 1 was turned on. The SNR was calculated by dividing the peak by the maximum value of the background in the cross correlation, the full width at half-maximum of the correlation function was about $1 \mathrm{~ns}$, and the distance resolution of the PR coding method was $15 \mathrm{~cm}$. The flight time was 120.28 ns. Then, we turned on all eight DFB lasers, and the correlation function of DFB laser 1 is shown in the Fig. 2(h). The SNR of the cross-correlation peak was getting a little worse due to the influence of the other laser echoes, which was still high enough with the accumulated time of $1 \mathrm{~s}$. The flight time of the cross-correlation peak was $120.27 \mathrm{~ns}$, which was nearly the same as the result when DFB laser 1 was turned on alone. The flight time of each laser beam will not be affected by the other channels. As Fig. 3 shows, all cross-correlation peaks were correctly calculated when all eight DFB lasers were turned on. When DFB lasers 1,3 , and 5 were turned off, there were no cross-correlation peaks appearing in the cross correlation of channels 1,3 , and 5 . The calculation results of each channel were independent of each other. Therefore, the PR multiplexing method can be applied to multi-beam single-photon measurement. 
(a)

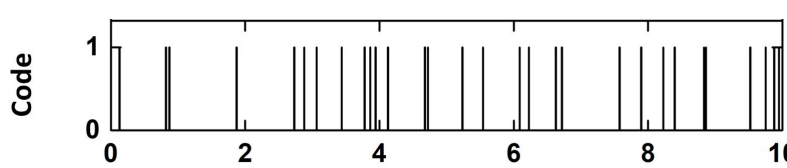

(b)

(c)

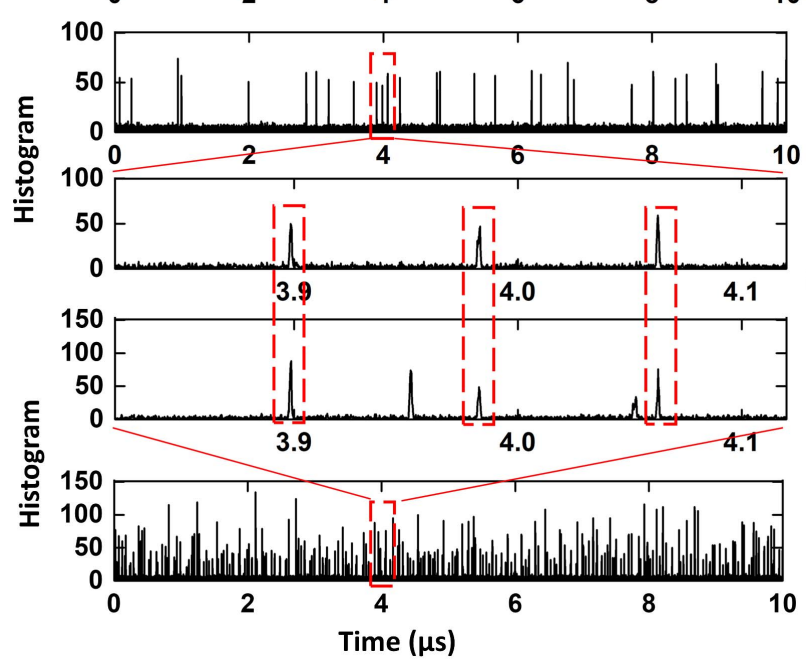

(f)

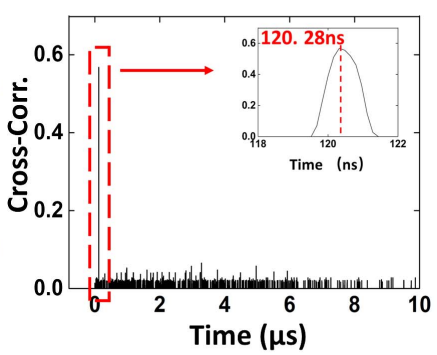

(g)

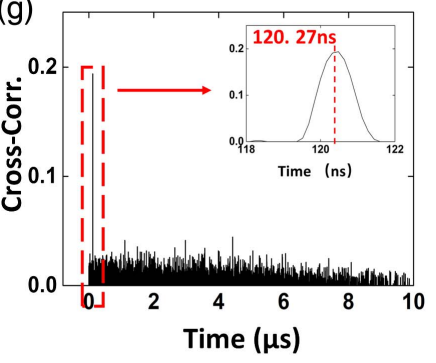

Fig. 2. (a) PR code 1. (b) The histogram of the detection events when only DFB laser 1 was turned on. (c) Subset of (b). (d) Subset of (e). (e) The histogram of the detection events when all of the lasers were turned on. (f) The cross correlation (Cross-Corr.) of the histogram in (b) with PR code 1. (g) The Cross-Corr. of the histogram in (e) with PR code 1.

(a)

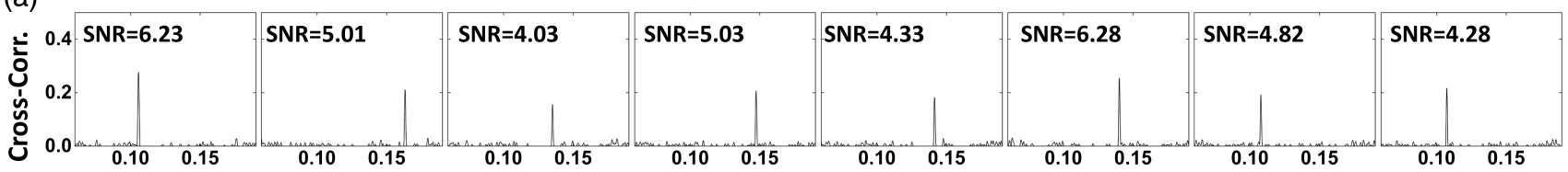

(b)

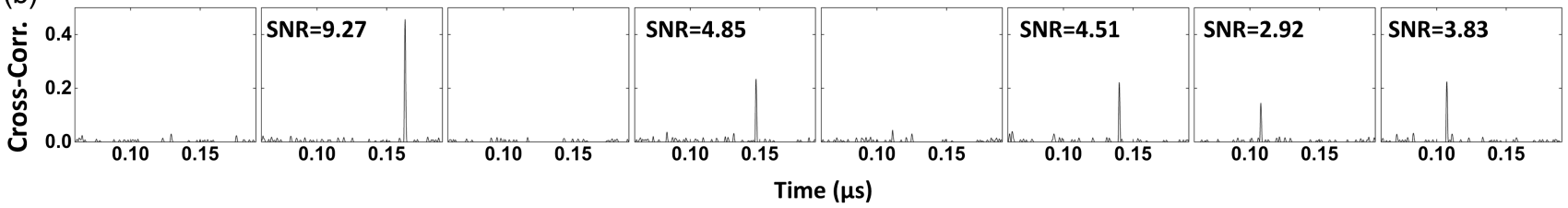

Fig. 3. (a) Cross-Corr. when all of the lasers were turned on. (b) Cross-Corr. when DFB lasers 2, 4, 6, 7, and 8 were turned on.

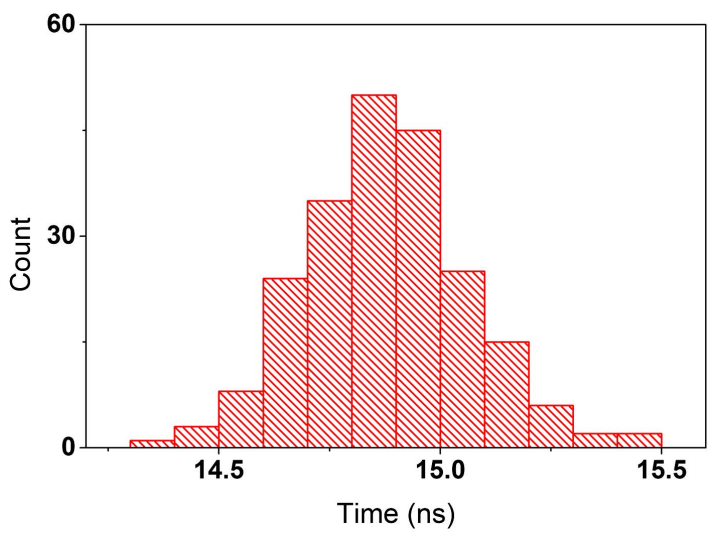

Fig. 4. TOF distribution between the 240 bits of ' 1 ' and their corresponding echo photons.
Furthermore, we analyzed the ranging error by measuring the distance between two targets, which could eliminate the effect of fixed delays in the laser emission and detection. The distance difference between the two targets was about $2 \mathrm{~m}$, and the flight time differences of the two targets measured by the eight-channel laser were $14.96 \mathrm{~ns}, 14.97 \mathrm{~ns}, 14.75 \mathrm{~ns}, 14.84 \mathrm{~ns}, 14.89 \mathrm{~ns}$, $14.86 \mathrm{~ns}, 14.88 \mathrm{~ns}$, and $14.78 \mathrm{~ns}$, respectively. The measurement error of each channel mainly came from the jitter between the synchronization signal and the PR codes generated by the FPGA. Figure 4 is the TOF distribution between the 240 bits of ' 1 ' and their corresponding echo photons. The average value of 240 sets of TOF data was $14.89 \mathrm{~ns}$, the root mean square error (RMSE) was $0.22 \mathrm{~ns}$, and the actual ranging accuracy of the system was $33 \mathrm{~mm}$. The error caused by FPGA could be eliminated by adding eight TDC channels to synchronously record eight PR codes for cross correlation. 


\section{Results and Discussions}

In the outdoor ranging experiments, the total output laser power of the EDFA was $12.9 \mathrm{~mW}$, when all eight lasers were turned on. The measurement targets were a white wall with an indoor distance of about $10 \mathrm{~m}$ and four outdoor buildings with different distances. We tested the system at night to avoid large environmental noise. We analyzed the ranging error by measuring the distance between the four buildings and the white wall. The average value of 240 sets of TOF data was taken as the real value

(a)

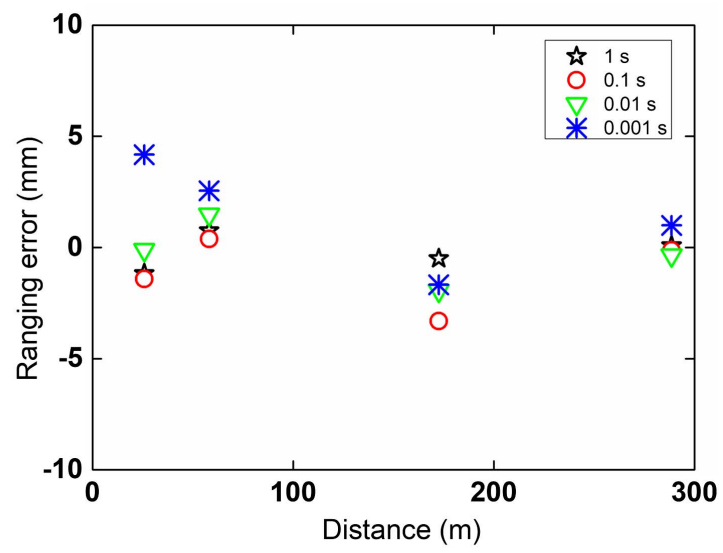

(b)

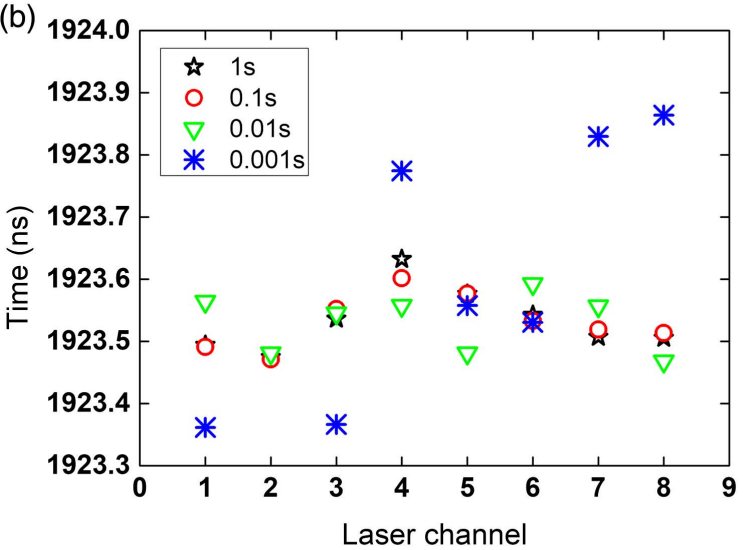

(c)

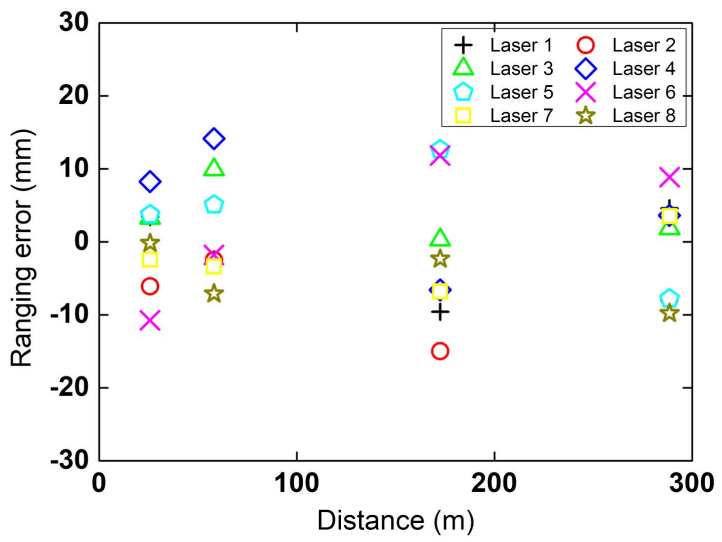

Fig. 5. (a) The average ranging error of eight channels with different accumulated times. (b) The flight time of each channel at the distance of $288.5 \mathrm{~m}$. (c) The ranging error of each channel with the accumulated time of $10 \mathrm{~ms}$. when the accumulated time was $1 \mathrm{~s}$. The photon count of the white wall was $3.3 \times 10^{6} \mathrm{cps}$. The distances between the four buildings and the white wall were about $25.7 \mathrm{~m}, 58.1 \mathrm{~m}$, $172.5 \mathrm{~m}$, and $288.5 \mathrm{~m}$, respectively. The photon counts of the detection signal of the four buildings were $3.59 \times 10^{6} \mathrm{cps}$, $2.57 \times 10^{6} \mathrm{cps}, 9.2 \times 10^{5} \mathrm{cps}$, and $6.6 \times 10^{5} \mathrm{cps}$, respectively. Figure 5(a) shows the ranging error between the average value of the eight-channel laser and the real value at each distance when the accumulated time was $1 \mathrm{~s}, 0.1 \mathrm{~s}, 0.01 \mathrm{~s}$, and $0.001 \mathrm{~s}$, respectively. The difference between the average value of eight channels and the real value was within $\pm 10 \mathrm{~mm}$. Figure 5(b) is the flight time of each channel at the distance of $288.5 \mathrm{~m}$ with different accumulated time. The real value of this distance was 1923.53 ns. The difference between the measured data of eight channels and the real value was all less than $0.1 \mathrm{~ns}$ when the accumulated time was no less than $10 \mathrm{~ms}$. Figure 5(c) is the difference between the flight time of eight channels and the real value when the accumulated time was $10 \mathrm{~ms}$. The ranging error of each channel was less than $20 \mathrm{~mm}$, as the relative distance was from $25.7 \mathrm{~m}$ to $288.5 \mathrm{~m}$.

When the accumulated time was reduced to $1 \mathrm{~ms}$, the ranging error of each channel at the distance of $288.5 \mathrm{~m}$ increases significantly, as shown in Fig. 5(b). Figure 6 is the measurement data. Comparing Figs. 6(a) and 6(b), it can be found that after the cumulative time was reduced from $10 \mathrm{~ms}$ to $1 \mathrm{~ms}$, the photon count of each echo was reduced to one, which was similar with the noise counts. The SNR of the correlation function peak was low, as the noise cannot be filtered out, resulting in the increasing measurement error. The SNRs of the correlation function of channels 1,2 , and 3 were $1.31,0.65$, and 1.85 , while their effective photon counts were 14,9 , and 16 , respectively. The total
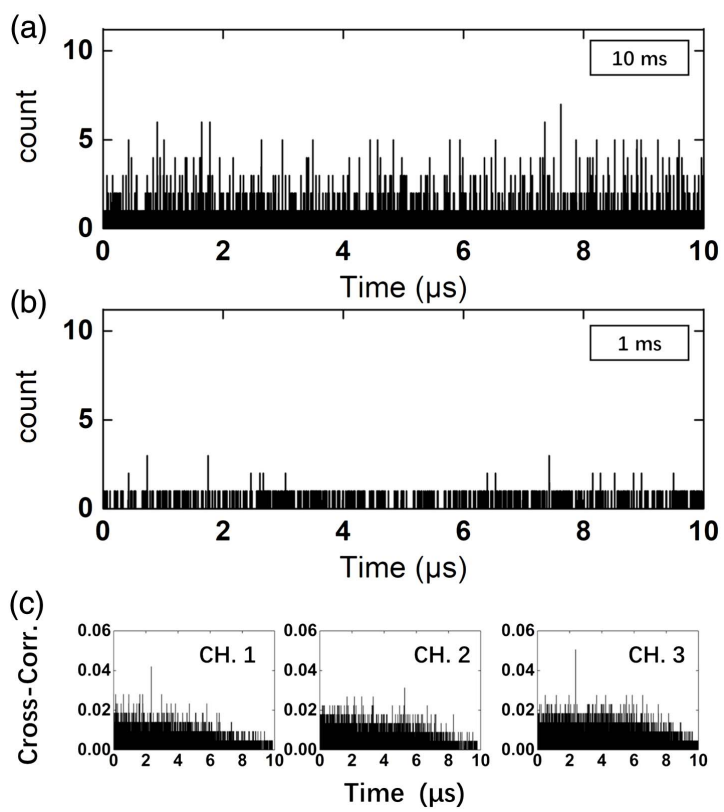

Fig. 6. (a) The histogram at the distance of $288.5 \mathrm{~m}$ when the accumulated time was $10 \mathrm{~ms}$. (b) The histogram at the distance of $288.5 \mathrm{~m}$ when the accumulated time was $1 \mathrm{~ms}$. (c) The Cross-Corr. function of laser channels 1, 2, and 3 when the accumulated time was $1 \mathrm{~ms}$. 
(a)

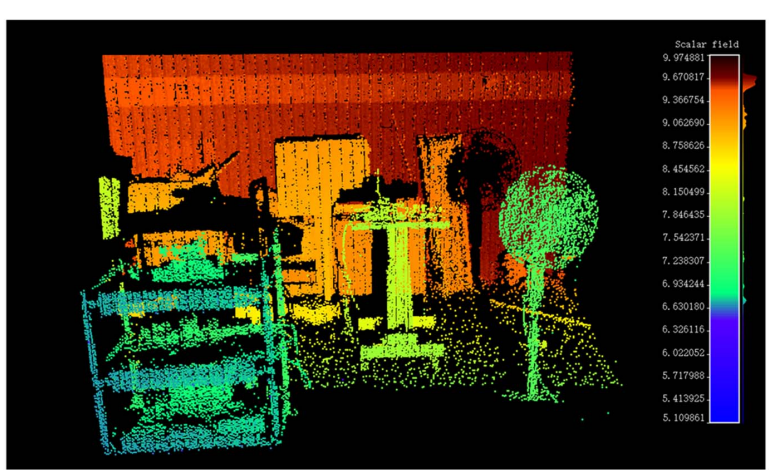

(b)

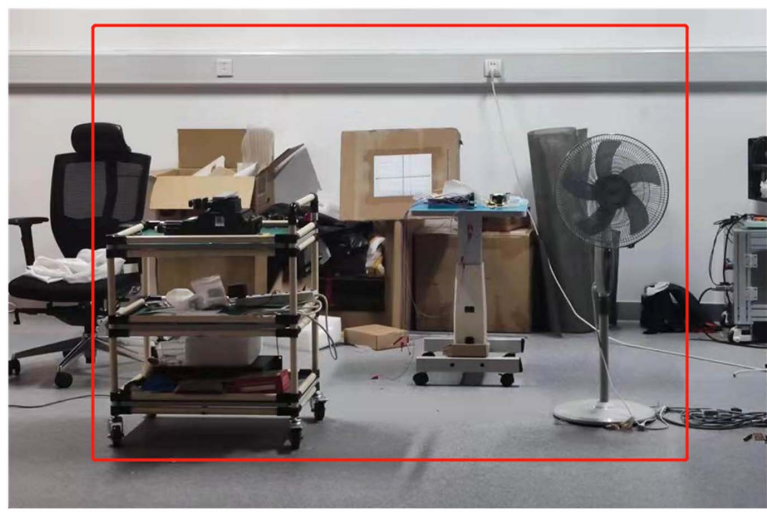

Fig. 7. (a) Reconstructed 3D image of the objects. (b) The picture of the objects.

photon count was 628 . The cross-correlation peaks of channels 1 and 3 still can be automatically identified, while their effective photon counts were only $\sim 1 / 40$ of the background.

We placed the experimental device on a two-dimensional rotating motor for scanning measurement in the case of a few returning photons and used these eight sets of $\mathrm{PR}$ codes to modulate eight DFB lasers with wavelengths of C34, C36,..., C48 (1550.12-1538.98 nm), referring to the measurement optical path in the previous work ${ }^{[25]}$. A blazed grating was placed at the laser emitting end, which was used to split the eight-wavelength coupled light into a $1 \times 8$ beam array with a divergence angle of $1 \mathrm{mrad}$. The measurement object was a range of $3 \mathrm{~m} \times$ $3 \mathrm{~m} \times 2 \mathrm{~m}$ at $10 \mathrm{~m}$ indoors. The horizontal movement of the motor was $8 \mathrm{mrad} / \mathrm{step}$, the number of steps was set to 41 , the vertical movement was $1 \mathrm{mrad} / \mathrm{step}$, and the number of steps was set to 200 . The total output optical power was $1 \mathrm{~mW}$, and the cumulative time was $1 \mathrm{~ms}$. The total photon count of the white wall $10 \mathrm{~m}$ away was less than 2000, with the maximum photon count of each echo less than five. Figure 7 is the reconstructed 3D image of the object and the actual picture of the object. The pixel of the reconstructed 3D image was $200 \times(41 \times 8)$. The electric fan, lifting platform, and three-layer cart in the physical picture were all well reproduced.

\section{Summary}

We demonstrated a multi-channel PR coding single-photon ranging system. It multiplexed multiple PR codes to simplify the detection system. Compared with the traditional TDM technique, it will not shorten the maximum unambiguous range while increasing the number of the channels. Eight PR code modulated lasers were coupled into one beam through a DWDM, and their echoes were detected only by one SPD and processing circuit. The crosstalk among the eight channels was very low, which can be ignored. The ranging error was less than $\pm 2.0 \mathrm{~cm}$. In the future, it can be eliminated through recording the PR codes at the output of the FPGA in real time to reduce the error to less than $\pm 5 \mathrm{~mm}$. The system is used to measure a target with a range of $3 \mathrm{~m}$ within $10 \mathrm{~m}$. When the number of returning photons was less than 2000 , as well as the maximum photon count of each echo being less than five, it still has a good range resolution capability. In multi-beam single-photon Lidar, the PR multiplexing technique can replace the TDM technique to realize long-distance ranging and imaging.

\section{Acknowledgement}

This work was supported by the National Natural Science Foundation of China (Nos. 11804099, 62075062, 11774095, and 11621404) and the Research Funds of Happiness Flower ECNU (No. 2021ST2110).

\section{References}

1. P. Du, F. Zhang, Z. Li, Q. Liu, M. Gong, and X. Fu, "Single-photon detection approach for autonomous vehicles sensing," IEEE Trans. Veh. Technol. 69, 6067 (2020).

2. D. Bronzi, Y. Zou, F. Villa, S. Tisa, A. Tosi, and F. Zappa, “Automotive threedimensional vision through a single-photon counting SPAD camera," IEEE T. Intell. Transp. 17, 782 (2016)

3. J. Rapp, J. Tachella, Y. Altmann, S. McLaughlin, and V. K. Goyal, “Advances in single-photon lidar for autonomous vehicles: working principles, challenges, and recent advances," IEEE Signal. Proc. Mag. 37, 62 (2020).

4. H. Riris, J. F. Cavanaugh, X. Sun, L. Ramos-Izquierdo, P. Liiva, M. Rodriguez, S. Schmidt, J. McGarry, C. Peters, G. B. Jackson, and D. E. Smith, "The lunar orbiter laser altimeter (LOLA) on NASA's lunar reconnaissance orbiter (LRO) mission," in Conference on Lasers and Electro-Optics/International Quantum Electronics Conference (Optical Society of America, 2009), pape CFJ1.

5. L. Irwin, N. C. Coops, M. Queinnec, G. McCartney, and J. C. White, "Single photon lidar signal attenuation under boreal forest conditions," Remote Sens. Lett. 12, 1049 (2021).

6. K. Y. Shrestha, W. E. Carter, K. C. Slatton, and T. K. Cossio, "Shallow bathymetric mapping via multistop single photoelectron sensitivity laser ranging," IEEE Trans. Geosci. Remote Sens. 50, 4771 (2012).

7. S. Chen, D. Liu, W. Zhang, L. You, Y. He, W. Zhang, X. Yang, G. Wu, M. Ren, H. Zeng, Z. Wang, X. Xie, and M. Jiang, “Time-of-flight laser ranging and imaging at $1550 \mathrm{~nm}$ using low-jitter superconducting nanowire singlephoton detection system," Appl. Opt. 52, 3241 (2013).

8. R. E. Warburton, A. McCarthy, A. M. Wallace, S. H. Marin, S. Cova, R. A. Lamb, and G. S. Buller, "Enhanced performance photon-counting time-of-flight sensor," Opt. Express 15, 423 (2007).

9. A. McCarthy, R. J. Collins, N. J. Krichel, V. Fernández, A. M. Wallace, and G. S. Buller, "Long-range time-of-flight scanning sensor based on highspeed time-correlated single-photon counting," Appl. Opt. 48, 6241 (2009).

10. A. Hou, Y. Hu, N. Zhao, J. Fang, S. Xu, and Q. Zhou, "Full-waveform fast correction method for photon counting lidar," Chin. Opt. Lett. 19, 052701 (2021). 
11. Y. Wang, L. Wang, C. Wu, Z. Li, L. Yang, and G. Wu, "Ultra-low detection delay drift caused by the temperature variation in a Si-avalanchephotodiode-based single-photon detector," Chin. Opt. Lett. 19, 082502 (2021).

12. R. Cheng, X. Guo, X. Ma, L. Fan, K. Fong, M. Poot, and H. Tang, "Self-aligned multi-channel superconducting nanowire single-photon detectors," Opt. Express 24, 27070 (2016).

13. R. Shu, G. Huang, L. Hou, Z. He, and Y. Hu, "Multi-channel photon counting three-dimensional imaging laser radar system using fiber array coupled Geiger-mode avalanche photodiode," Proc. SPIE 8542, 85420C (2012).

14. A. Cuccato, S. Antonioli, M. Crotti, I. Labanca, A. Gulinatti, I. Rech, and M. Ghioni, "Complete and compact 32-channel system for time-correlated single-photon counting measurements," IEEE Photonics. J. 5, 6801514 (2013).

15. Z. Li, E. Wu, C. Pang, B. Du, Y. Tao, H. Peng, H. Zeng, and G. Wu, "Multibeam single-photon-counting three-dimensional imaging lidar," Opt. Express 25, 10189 (2017).

16. R. Brown, P. Hartzell, and C. Glennie, "Evaluation of SPL100 single photon lidar data," Remote Sens. 12, 722 (2020).

17. Y. Jiang, S. Karpf, and B. Jalali, "Time-stretch LiDAR as a spectrally scanned time-of-flight ranging camera," Nat. Photonics 14, 14 (2020).
18. R. E. Warburton, F. Izdebski, C. Reimer, J. Leach, D. G. Ireland, M. Padgett, and G. S. Buller, "Single-photon position to time multiplexing using a fiber array," Opt. Express 19, 2670 (2011).

19. T. Zheng, G. Shen, Z. Li, L. Yang, H. Zhang, E. Wu, and G. Wu, "Frequencymultiplexing photon-counting multi-beam LiDAR," Photon. Res 7, 1381 (2019).

20. X. Sun and J. B. Abshire, "Modified PN code laser modulation technique for laser measurements," Proc. SPIE 7199, 71990P (2009).

21. M. Matsumoto and T. Nishimura, "Mersenne twister: a 623-dimensionally equidistributed uniform pseudo-random number generator," ACM Trans. Model. Comput. Simul. 8, 3 (1998).

22. D. R. Cremons, X. Sun, J. B. Abshire, and E. Mazarico, "Small PN-code lidar for asteroid and comet missions-receiver processing and performance simulations," Remote Sens. 13, 2282 (2021).

23. T. Lunghi, C. Barreiro, O. Guinnard, R. Houlmann, X. Jiang, M. A. Itzler, and H. Zbinden, "Free-running single-photon detection based on a negative feedback InGaAs APD," J. Mod. Opt. 59, 1481 (2012).

24. Q. Zhang, L. Chen, and N. Chen, "Pseudo-random single photon counting: a high-speed implementation," Biomed. Opt. Express 1, 41 (2010).

25. D. Wu, T. Zheng, L. Wang, X. Chen, L. Yang, Z. Li, and G. Wu, "Multi-beam single-photon LiDAR with hybrid multiplexing in wavelength and time," OPT Laser Technol. 145, 107477 (2022). 